\title{
The effect of heat stress on frame switch splicing of X-box binding protein 1 gene in horse
}

\author{
Hyo Gun Lee', Saichit Khummuang', Hyun-Hee Youn', Jeong-Woong Park', Jae-Young Choi', \\ Teak-Soon Shin', Seong-Keun Cho', Byeong-Woo Kim', Jakyeom Seo', Myunghoo Kim', \\ Tae Sub Park', and Byung-Wook Cho ${ }^{1, *}$
}

\section{* Corresponding Author: Byung-Wook Cho Tel: +82-55-350-5515, Fax: +82-55-350-5519, E-mail: bwcho@pusan.ac.kr}

${ }^{1}$ Department of Animal Science, College of Natural Resources and Life Sciences, Pusan National University, Miryang 50463, Korea

${ }^{2}$ Graduate School of International Agricultural Technology and Institute of Green-Bio Science and Technology, Seoul National University, Pyeongchang 25354, Korea

ORCID

Hyo Gun Lee

https://orcid.org/0000-0002-3286-7368 Saichit Khummuang

https://orcid.org/0000-0002-8819-3964 Hyun-Hee Youn

https://orcid.org/0000-0002-8169-4213 Jeong-Woong Park

https://orcid.org/0000-0003-0885-3078 Jae-Young Choi

https://orcid.org/0000-0001-8264-1482 Teak-Soon Shin

https://orcid.org/0000-0001-5362-9206 Seong-Keun Cho

https://orcid.org/0000-0001-9309-2327

Byeong-Woo Kim

https://orcid.org/0000-0003-3300-0173

Jakyeom Seo

https://orcid.org/0000-0002-9176-5206

Myunghoo Kim

https://orcid.org/0000-0002-8444-6952

Tae Sub Park

https://orcid.org/0000-0002-0372-5467

Byung-Wook Cho

https://orcid.org/0000-0002-7739-1391

Submitted Oct 11, 2018; Revised Nov 26, 2018; Accepted Dec 15, 2018
Objective: Among stress responses, the unfolded protein response (UPR) is a well-known mechanism related to endoplasmic reticulum (ER) stress. ER stress is induced by a variety of external and environmental factors such as starvation, ischemia, hypoxia, oxidative stress, and heat stress. Inositol requiring enzyme $1 \alpha$ (IRE1 $1 \alpha)$-X-box protein 1 (XBP1) is the most conserved pathway involved in the UPR and is the main component that mediates IRE1 $\alpha$ signalling to downstream ER-associated degradation (ERAD)- or UPR-related genes. XBP1 is a transcription factor synthesised via a novel mechanism called 'frame switch splicing', and this process has not yet been studied in the horse XBP1 gene. Therefore, the aim of this study was to confirm the frame switch splicing of horse $X B P 1$ and characterise its dynamics using Thoroughbred muscle cells exposed to heat stress.

Methods: Primary horse muscle cells were used to investigate heat stress-induced frame switch splicing of horse XBP1. Frame switch splicing was confirmed by sequencing analysis. XBP1 amino acid sequences and promoter sequences of various species were aligned to confirm the sequence homology and to find conserved cis-acting elements, respectively. The expression of the potential XBP1 downstream genes were analysed by quantitative real-time polymerase chain reaction.

Results: We confirmed that splicing of horse XBP1 mRNA was affected by the duration of thermal stress. Twenty-six nucleotides in the mRNA of $X B P 1$ were deleted after heat stress. The protein sequence and the cis-regulatory elements on the promoter of horse $X B P 1$ are highly conserved among the mammals. Induction of putative downstream genes of horse XBP1 was dependent on the duration of heat stress. We confirmed that both the mechanisms of XBP1 frame switch splicing and various binding elements found in downstream gene promoters are highly evolutionarily conserved.

Conclusion: The frame switch splicing of horse XBP1 and its dynamics were highly conserved among species. These results facilitate studies of ER-stress in horse.

Keywords: Thoroughbred; Heat Stress; X-box Binding Protein 1; Quantitative Real-time Polymerase Chain Reaction (qRT-PCR)

\section{INTRODUCTION}

Organisms have evolved impressive mechanisms to mitigate the negative effects of external stressors, including the unfolded protein response (UPR). The UPR is a well- known mechanism induced by endoplasmic reticulum (ER)-stress. When an organism is exposed to ER stress, UPR-related pathways are activated by three sensor proteins which exist in the ER: activating transcription factor-6 (ATF6), protein kinase R (PKR)-like endoplasmic reticulum kinase (PERK), and inositol requiring enzyme $1 \alpha$ (IRE1 $\alpha)$ [1]. Binding immunoglobulin protein (BiP), also known as $78 \mathrm{kDa}$ glucose-regulated protein (GRP78), is a lumen-resident 
molecular chaperone which is markedly up-regulated under ER-stress, dissociating from the three sensor proteins to activate each UPR pathway when unfolded and misfolded proteins are accumulated in the ER.

Among them, IRE1 $\alpha$-X-box protein 1 (XBP1) is the most conserved machinery utilised in the UPR. IRE1 $\alpha$ is a transmembrane protein which self-activates via homo-dimerization and transphosphorylation during the UPR. Activated IRE1 $\alpha$ functions similarly to endoribonuclease facilitating the splicing of XBP1 mRNA in the cytosol [2,3]. This form of splicing is unconventional, as most pre-mRNAs are frequently the subject of spliceosome-dependent splicing in the nucleus [4]. Mammalian XBP1 mRNA contains two characteristic stemloop structures in which IRE1a-dependent splicing occurs, removing 26 nucleotides and exposing the transcriptional activation domain. As a consequence, translation of the spliced $X B P 1$ ( $(X B P 1)$ produces a longer protein which functions as a transcription factor [3]. This novel splicing mechanism is called 'frame switch splicing' to distinguish it from conventional splicing occurring within the nucleus [5]. In contrast to the un-spliced form of XBP1 (usXBP1), sXBP1 is translocated into the nucleus following synthesis, where it induces the upregulation of genes related to ER-associated degradation (ERAD). ER homeostasis is thus maintained by the refolding and degradation of unfolded or misfolded proteins by processes facilitated by the IRE1 $\alpha-X B P 1$ pathway.

The DNA-binding properties of sXBP1 are also important in the mediation of proper UPR function under the conditions of ER-stress. There are four evolutionarily conserved binding sites which can be found in the promoter region of UPR related genes, known as: ER stress response element (ERSE, consensus sequence: CCAAT-N9-CCACG), UPR element (UPRE, consensus sequence: TGACGTGG/A), ERSEII (consensus sequence: ATTGG-N1-CCACGT), and UPREII (consensus sequence ATTGGTCCGCGT). Among these elements, ERSE has been well studied so as to ascertain the exact function of XBP1. The consensus sequence of ERSE is CCAAT-N9-CCACG [6,7]. Both ATF6 and XBP1, two basic leucine zipper proteins related to the UPR, can bind to the CCACG region of ERSE following the binding of NF-Y to the CCAAT region $[3,8]$. In response to ER stress, ER chaperone genes are transcriptionally regulated by the ATF6 and IRE1 $\alpha-$ $\mathrm{XBP} 1$ pathways. ERSE is also found in the promoter region of $X B P 1$, suggesting that positive feedback of $X B P 1$ transcription can sustain the function of XBP1 during the UPR.

Thoroughbred is one of the most adaptive horse breeds when considering racing performance, and is commonly used as a representative exercise model in the field of exercise physiology. The muscles of horses are subjected to higher thermal stress after exercise than other tissues [9], and although it is known that ER-stress is induced by heat stress, the horse XBP1 gene has not yet been studied. Thus, the purpose of this study is to investigate whether the horse $X B P 1$ gene is evolutionarily conserved and to characterise its dynamics using Thoroughbred muscle cells exposed to heat stress.

\section{MATERIALS AND METHODS}

\section{Tissue sampling}

A skeletal muscle tissue biopsy was performed on the leg of a neonatal Thoroughbred to allow the cultivation of horse muscle cells. All materials and methods were approved by the Pusan National University-Institutional Animal Care and Use Committee (Approval Number: PNU-2015-0864).

\section{Primary horse muscle cell culture and heat stress}

Horse muscle cells were maintained and sub-passaged in DMEM (Gibco, Grand Island, NY, USA) and were supplemented with $10 \%$ foetal bovine serum (Invitrogen, Carlsbad, CA, USA) and $1 \%$ antibiotic-antimycotic (ABAM; Invitrogen, USA). The cells were cultured in a humidified atmosphere with $5 \% \mathrm{CO}_{2}$ at $37^{\circ} \mathrm{C}$. Routine fluid renewals were performed 3 times per week. At $70 \%$ to $80 \%$ confluence, cells were gently washed twice with phosphate-buffered saline and harvested using $0.05 \%$ trypsin-ethylenediaminetetraacetic acid (Welgene, Gyeongsan, Korea) for total RNA extraction. To apply heat shock, the $70 \%$ to $80 \%$ confluent horse muscle cells were transferred to an incubator at a temperature of $40^{\circ} \mathrm{C}$ for $1 \mathrm{~h}$ and $4 \mathrm{~h}$ followed by a $4 \mathrm{~h}$ recovery period in an atmosphere of $5 \% \mathrm{CO}_{2}$.

\section{RNA extraction and cDNA synthesis}

Total RNA was extracted from horse muscle cells using TRIzol (Invitrogen, Karlsruhe, Germany) according to the manufacturer's instructions. The purity of the extracted RNA was confirmed by measuring absorbance at $230 \mathrm{~nm}$ and $260 \mathrm{~nm}$ using a spectrophotometer (ND-1000, Nanodrop Technologies Inc., Wilmington, DE, USA), and RNA with a purity (OD value of $230 \mathrm{~nm} / 260 \mathrm{~nm}$ ) greater than 1.8 was selected for further analysis and stored at $-70^{\circ} \mathrm{C}$ until the experiment was carried out.

To synthesise cDNA, $1 \mu \mathrm{g}$ of RNA and $1 \mu \mathrm{L}$ each of oligodT (Invitrogen, Waltham, MA, USA) and RNase-Free Water were added. The RNA was denatured at $80^{\circ} \mathrm{C}$ for $3 \mathrm{~min}$ and cDNA was synthesised using $4 \mu \mathrm{L}$ of $5 \times \mathrm{RT}$ (reverse transcription) buffer, $5 \mu \mathrm{L}$ of $2 \mathrm{mmol} / \mathrm{L}$ dNTPs, $0.5 \mu \mathrm{L}$ of RNase inhibitor, and $1 \mu \mathrm{L}$ of M-MLV (Moloney-murine leukaemia virus) RT (Promega, Madison, WI, USA).

\section{Reverse transcription polymerase chain reaction and} real time-quantitative polymerase chain reaction The NCBI (http://www.ncbi.nlm.nih.gov) and the Ensembl Genome Browser (www.ensembl.org) were utilised to retrieve gene sequence information. The primers used in the amplifi- 
cation of the genes (Table 1) were synthesised using the PRIMER3 software (http://bioinfo.ut.ee/primer3-0.4.0/). Reverse transcription polymerase chain reaction (RT-PCR) and real-time quantitative PCR (qPCR) were carried out using a C1000 Thermal Cycler (Bio Rad, Hercules, CA, USA) using $25 \mu \mathrm{L}$ of reaction solution to measure the relevant expression of target genes. The solution was created as follows: $2 \mu \mathrm{L}$ of diluted cDNA (50 ng/ $\mu \mathrm{L}$ ) was added to $14 \mu \mathrm{L}$ of SYBR green master mix (Bio Rad, USA) and $1 \mu \mathrm{L}$ each of $5 \mathrm{pmol} / \mu \mathrm{L}$ diluted forward and reverse primers. The conditions used for the realtime qPCR were as follows: initial denaturation at $94^{\circ} \mathrm{C}$ for 10 mins followed by 40 cycles of denaturation at $94^{\circ} \mathrm{C}$ for $10 \mathrm{~s}$, annealing at $60^{\circ} \mathrm{C}$ for $10 \mathrm{~s}$, and extension at $72^{\circ} \mathrm{C}$ for $30 \mathrm{~s}$. All measurements were carried out in triplicate for each specimen, and the $2^{-\Delta \Delta C t}$ method [10] was used to determine relative gene expression. The relative expression of target genes was normalised with glyceraldehyde 3-phosphate dehydrogenase.

\section{Sequencing}

Agarose gel electrophoresis was performed using the PCR products amplified by the XBP1 primer set (Forward: $5^{\prime}$-AGC TCGAATGAGTGAGCTGG-3', reverse: 5'-ATCCATGGGG AGAGGTTCTGG-3') (Table 1). The clear PCR bands were then removed from the gel using a LaboPass gel extraction kit (COSMO GENETECH, Seoul, Korea). The amplicons were ligated using the pGEM-T easy vector (Promega, USA) for DNA sequencing with T7 (5'-TAATACGACTCACTATA-3') and SP6 (5'-CTATAGTGTCACCTAAAT-3') primers. Se-

Table 1. Primer sets used in this study

\begin{tabular}{llcc}
\hline $\begin{array}{c}\text { Primer } \\
\text { name }\end{array}$ & \multicolumn{1}{c}{ Primer sequence (5' to 3') } & Tm ( $\left.{ }^{\circ} \mathbf{C}\right)$ & $\begin{array}{c}\text { Product } \\
\text { size (bp) }\end{array}$ \\
\hline Hsp72 F & CGACCTCAACAAGAGCATCA & 60 & 213 \\
Hsp72 R & AAGATCTGCGTCTGCTTGGT & & \\
Bip F & TCTCTGAGACCCTGACTCGG & 58 & 217 \\
Bip R & ATCTGGGTTTATGCCACGGG & & \\
EDEM1 F & TCTGTGGACAAGCACCTTCG & 58 & 232 \\
EDEM1 R & GGAGAGCCGGTCAGATCAAG & & \\
GRP94 F & CTCCTGCGATCAGAAGGGAC & 58 & 120 \\
GRP94 R & TCGACTTCATCGTCAGCTCG & & \\
HRD1 F & GTGATGGGCAAGGTGTTCTT & 58 & 274 \\
HRD1 R & GGATGCCCAAGAGGAACATA & & \\
XBP1 F & AGCTCGAATGAGTGAGCTGG & 58 & 294 \\
XBP1 R & ATCCATGGGGAGAGGTTCTGG & & \\
sXBP1 F & GTCTGCTGAGTCCGCAGCAGGT & 58 & 209 \\
sXBP1 R & TGGGTCCTTCTGGGTGACC & & \\
usXBP1 F & GCAGCACTCAGTCTACGTGCG & 58 & 275 \\
usXBP1 R & CAGCTTGGCTGATGACGTCCC & & \\
GAPDH F & GGTGAAGGTCGGAGTAAACG & 60 & 106 \\
GAPDH R & AATGAAGGGGTCATTGATGG & & \\
\hline
\end{tabular}

Hsp, heat shock protein; Bip, binding immunoglobulin protein; EDEM1, enhancing alpha-mannosidase-like protein 1; GRP, glucose-regulated protein; HRD1, E3 ubiquitin-protein ligase synoviolin; XBP1, X-box protein 1; sXBP1, spliced XBP1; usXBP1, un-spliced XBP1; GAPDH, glyceraldehyde 3-phosphate dehydrogenase. quencing results were analysed against annotated horse genomes using the basic local alignment search tool (BLAST).

\section{Secondary structure analysis}

Secondary structure prediction of the deleted nucleotides in XBP1 mRNA was performed using Geneious version (6.0.6) (http://www.geneious.com) [11]. The predicted RNA folding structures were created using Turner's energy model at $37^{\circ} \mathrm{C}$.

\section{Alignment of protein and promoter sequences}

The XBP1 protein sequences of the different species including homo sapiens (ENSG00000100219), equus caballus (ENSECAG 00000014780), mus musculus (ENSMUSG00000020484), gallus gallus (ENSGALG00000005796), xenopus laevis (ENSX ETG00000027416), and danio rerio (ENSDARG00000035622) were retrieved from Ensembl release 92 (http://asia.ensembl. org) and aligned using Geneious version (6.0.6) (http://www. geneious.com) [11]. Approximately 200 upstream nucleotides from the transcription start site of XBP1 in the five species (homo sapiens, macaca mulatta, equus caballus, bos taurus, and mus musculus) were retrieved from the NCBI database. Putative transcription factors, which bind to the promoter of $X B P 1$, were annotated using EMBOSS: tfscan based on the TRANSFAC database. The upstream sequences of horse BiP, GRP94, and homocysteine-responsive endoplasmic reticulumresident ubiquitin-like domain member 1 protein (Herpud1) were retrieved to search the binding sites of XBP1.

\section{Statistical analysis}

Means and standard deviations were calculated using Microsoft Excel. The statistical significance of the results $\left({ }^{*} p<0.05\right.$, ${ }^{* *} \mathrm{p}<0.01$, or $\left.{ }^{* * *} \mathrm{p}<0.001\right)$ was assessed using one-way analysis of variance followed by post-hoc comparison (Tukey's honestly significant difference test) using the Prism 5 program (San Diego, CA, USA).

\section{RESULTS}

Heat mediated splicing of $X B P 1$ in horse muscle cells To induce XBP1 splicing, horse muscle cells were subjected to a $1 \mathrm{~h}$ and $4 \mathrm{~h}$ heat shock at $40^{\circ} \mathrm{C}$ followed by $4 \mathrm{~h}$ of recovery at $37^{\circ} \mathrm{C}[12] . H s p 72$, a member of the heat shock protein 70 family and a chaperone protein used as a marker of heat stress, immediately increased during the $1 \mathrm{~h}$ heat shock, and increased significantly during the $4 \mathrm{~h}$ heat shock. $H s p 72$ in cells subjected to the $4 \mathrm{~h}$ heat shock subsequently decreased during the $4 \mathrm{~h}$ recovery period (Figure $1 \mathrm{~A}$ ). To detect the $X B P 1$ splicing site, we predicted the deleted region based on the human $X B P 1$ splicing site and designed a primer set to produce a $294 \mathrm{bp}$ PCR amplicon encompassing exon 3 of horse $X B P 1$ (Figure 1B). As a result, we successfully detected a clear band in the 294 bp PCR product indicative of XBP1 splic- 
(A)

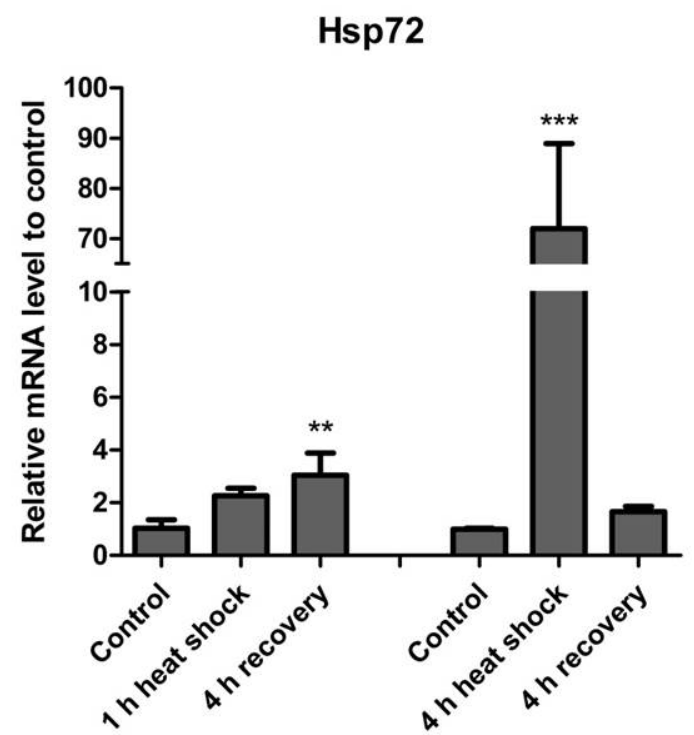

(B)

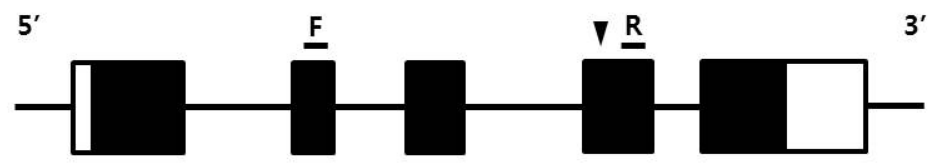

(C)

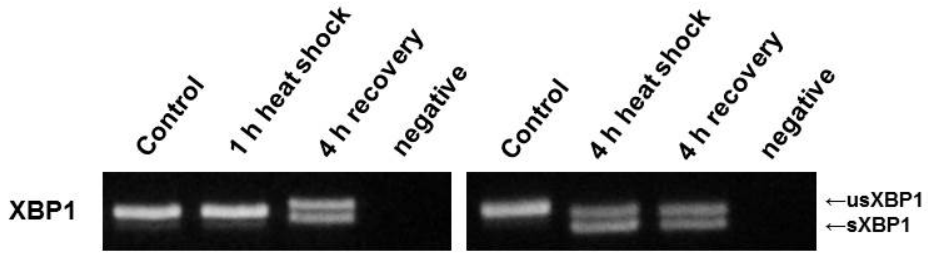

(D)

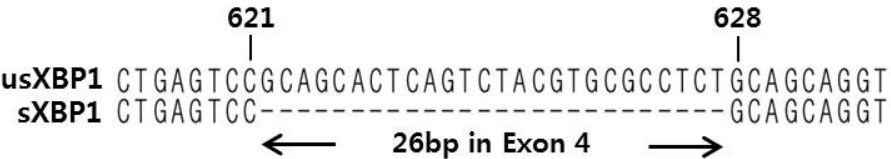

(E)

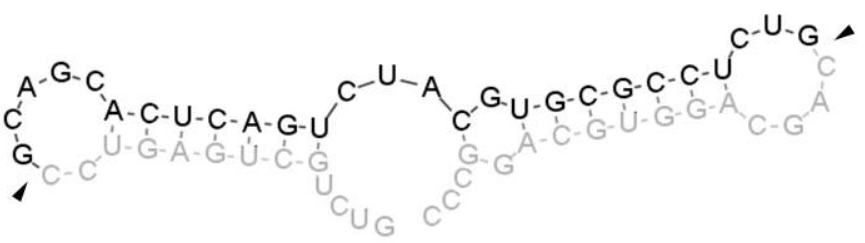

Figure 1. Heat stress mediated XBP1 splicing in Thoroughbred muscle derived cells. (A) Time course induction of Hsp72. Horse muscle cells were incubated for $1 \mathrm{~h}$ and $4 \mathrm{~h}$ at $40^{\circ} \mathrm{C}$ followed by $4 \mathrm{~h}$ recovery shock at $37^{\circ} \mathrm{C}$. Hsp72 expression was analysed by real-time qPCR using cDNA after both the $1 \mathrm{~h}$ heat shock (one-way ANOVA, $\mathrm{p}=0.011$ ) and $4 \mathrm{~h}$ heat shock (one-way ANOVA, $p=0.0002$ ). Asterisk denotes statistical significance ( ${ }^{* *} p<0.01,{ }^{* *} p<0.001$ ) of post-hoc comparisons (Tukey's HSD) with the control for each heat shock duration. The results were normalised to GAPDH. Error bars represent the standard deviation $(n=3)$. (B) Schematic presentation of the horse XBP1 gene structure. White and black boxes indicate untranslated regions and exons, respectively. The location of the forward (F) and reverse (R) primers for detection of the spliced XBP1 mRNA are shown. The arrow head indicates the location of the spliced region relative to the primers. (C) XBP1 mRNA splicing after both the $1 \mathrm{~h}$ and $4 \mathrm{~h}$ heat shock followed by a $4 \mathrm{~h}$ recovery period. RT-PCR was used to detect usXBP1 and sXBP1 mRNA. Upper bands (294 bp) and lower bands (268 bp) indicate usXBP1 and sXBP1, respectively. (D) Nucleotide sequences of cDNA corresponding to usXBP1 and SXBP1 transcripts around the splicing sites. 26 bp deletion occurred in exon 4 of XBP1 mRNA after heat shock. (E) The predicted secondary folding structure of the deleted region in the XBP1 mRNA at $37^{\circ} \mathrm{C}$ depicted by Turner's energy model. The cleavage sites in XBP1 mRNA are indicated by arrow heads. XBP1, X-box binding protein 1; Hsp72, heat shock $70 \mathrm{kDa}$ protein 1; qPCR, quantitative real-time polymerase chain reaction; ANOVA, analysis of variance; HSD, honestly significant difference; GAPDH, glyceraldehyde 3-phosphate dehydrogenase; RT-PCR, Reverse transcription polymerase chain reaction; usXBP1, un-spliced XBP1; SXBP1, spliced XBP1.

ing. Interestingly, in cells exposed to the $1 \mathrm{~h}$ heat shock, $X B P 1$ splicing was not induced during heat shock exposure but was induced during the $4 \mathrm{~h}$ recovery period. In cells exposed to the $4 \mathrm{~h}$ heat shock, $X B P 1$ splicing was clearly induced (Figure $1 C$ ). These results show that heat mediated XBP1 splicing in horse muscle cells is only induced by more than $1 \mathrm{~h}$ of heat shock and that the spliced form of XBP1 mRNA persists until after the $4 \mathrm{~h}$ recovery period. Next, we cloned the bands of both un-spliced (usXBP1) and spliced XBP1 (sXBP1) and conducted sequencing analysis to identify the splicing regions, the results of which showed that a region consisting of 26 nucleotides within exon 4 of horse XBP1 were deleted (Figure 1D). Furthermore, we predicted the secondary structure of $s X B P 1$ using the mRNA sequence from around the deleted region of $X B P 1$. The result shows that there are two characteristic loop structures defined within the conserved
IRE1 a cleavage sites [5] (Figure 1E). Taken together, the results of these analyses confirm that the horse XBP1 gene is subjected to frame shift splicing.

Heat stress-induced changes in the ratio of $s X B P 1$ to usXBP1 in horse muscle cells

To understand $X B P 1$ splicing during exposure to heat stress, we designed primer sets to specifically detect $u s X B P 1$ and $s X B P 1$ transcripts (Figure 2A). We confirmed the specificity of primer amplification by gel electrophoresis using the cDNA from horse muscle cells exposed to the $4 \mathrm{~h}$ heat shock (Figure $2 \mathrm{~B})$. Following the $1 \mathrm{~h}$ heat shock, the ratio of $s X B P 1$ transcripts to total $X B P 1$ transcripts slightly increased, and the ratio was significantly increased after the $4 \mathrm{~h}$ recovery period. Conversely, the ratio had significantly increased after the $4 \mathrm{~h}$ heat shock and remained high until the $4 \mathrm{~h}$ recovery period 
(A)

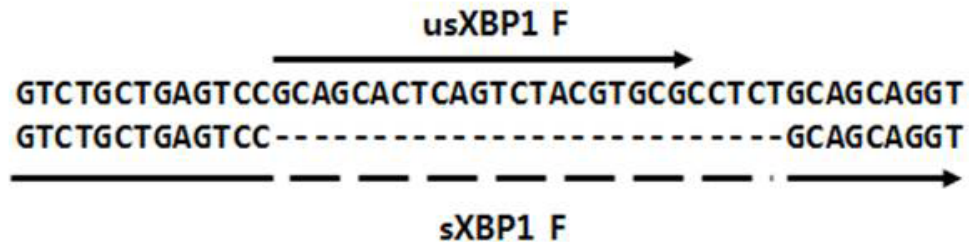

(B)

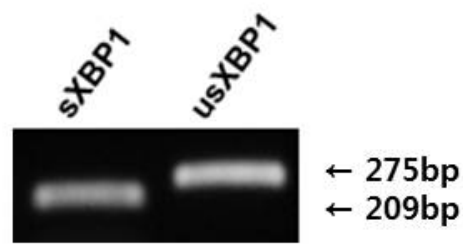

(C)
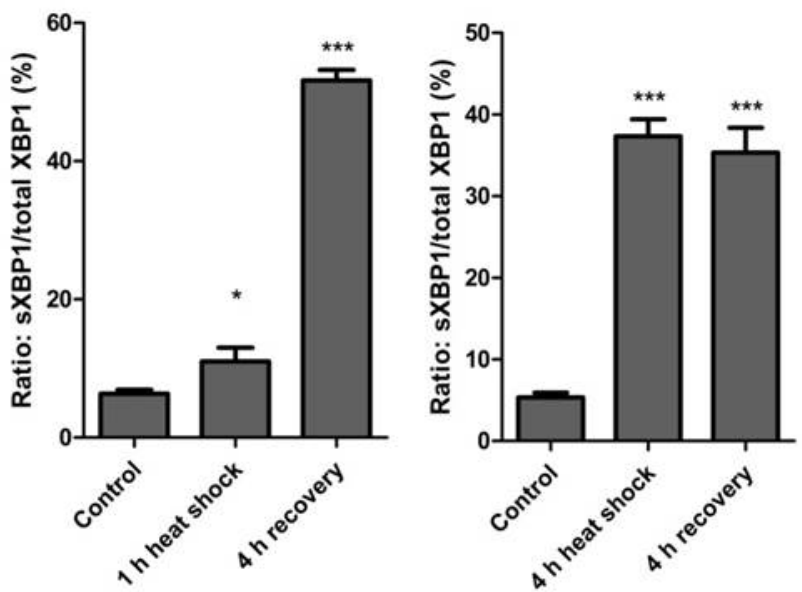

Figure 2. Expression of usXBP1 and sXBP1 mRNA after heat shock exposure. (A) Design of forward primers detecting the specific forms of XBP1 mRNA. Black arrows indicate each forward primer. Dotted line shows the deleted region of $s X B P 1$. (B) RT-PCR analysis for the confirmation of the primer sets detecting the specific forms of XBP1 mRNA. (C) The ratio of $s$ XPP1 to total XBP1 mRNA was evaluated by real-time qPCR of cDNA obtained after the $1 \mathrm{~h}$ heat shock $(\mathrm{n}=3$, one-way ANOVA, $\mathrm{p}<0.0001)$ and $4 \mathrm{~h}$ heat shock $(n=3$, one-way ANOVA, $p<0.0001)$. Asterisk denotes statistical significance $\left.{ }^{* *} p<0.01,{ }^{* * *} p<0.001\right)$ of post-hoc comparisons (Tukey's HSD) with the control for each heat shock duration. The results were normalised to GAPDH. Error bars represent the standard deviation $(n=3)$. XBP1, X-box binding protein 1; usXBP1, un-spliced XBP1; sXBP1, spliced XBP1; RT-PCR, Reverse transcription polymerase chain reaction; qPCR, quantitative real-time polymerase chain reaction; ANOVA, analysis of variance; HSD, honestly significant difference; GAPDH, glyceraldehyde 3-phosphate dehydrogenase.

(Figure 2C). These results indicate that prolonged heat stress affects XBP1 splicing.

\section{Sequence homology of horse XBP1}

The XBP1 protein sequence homology was investigated using human, horse, mouse, chicken, frog, and zebrafish sequences. Human usXBP1 and sXBP1 showed the highest similarity to horse usXBP1 and sXBP1 (usXBP1: 84.411\% and sXBP1: $85.039 \%$ ). Birds, amphibians, and fish showed relatively lower similarities in their XBP1 sequences than those seen in mammals (Figure 3A). Next, the human, monkey, horse, cattle, and mouse promoter sequences were aligned to search for conserved transcription factor binding elements. We found that the CCAAT box-binding transcription factor, ATF, and ERSE were highly conserved among taxonomic groups. Additionally, the binding sites for transcription factor II $D$ and specificity protein 1 in the horse $X B P 1$ promoter were predicted (Figure 3B).

According to a study of human $X B P 1$ genes, $s X B P 1$ encodes longer proteins than $u s X B P 1$, and the frame-shifted part of sXBP1 exhibits higher transcriptional activator activity [3].
When usXBP1 and sXBP1 were translated to identify the open reading frame, we noted that splicing of $X B P 1$ replaced the C-terminal portion of XBP1 with 210 amino acids. Using protein domain prediction tools on horse XBP1 protein sequences showed that the unchanged part of XBP1 contains a basic leucine zipper domain. We could predict any domain based on the C-terminal part of horse sXBP1 (Figure 3C), however, based on the protein sequence homology of horse sXBP1, it is reasonable to assume that the replaced part of the $\mathrm{C}$-terminal has a transcription activator domain. Taken together, these results indicate that not only the cis-regulatory elements in the promoter and protein sequence of XBP1, but also the protein domains of XBP1 are highly conserved among the studied groups.

\section{Expression of downstream genes of XBP1}

XBP1 contains several distinct binding elements relating to the promoter regions of several downstream genes, including ERSE, ERSE II, UPRE, and UPRE-II. BiP and GRP94 are two representative genes regulated by XBP1 [6,7]. Additionally, Herpud1, a molecular chaperone for proteins in the ER, con- 
(A)

usXBP1

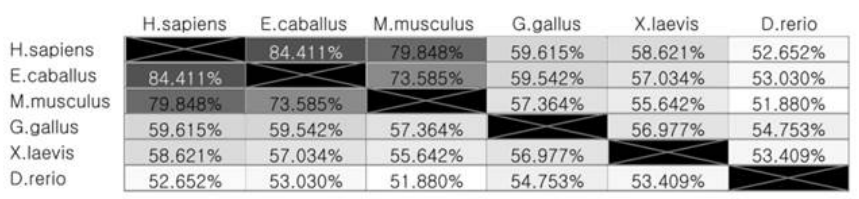

sXBP1

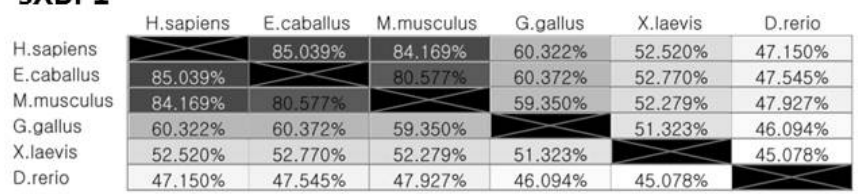

(B)

\begin{tabular}{|c|c|}
\hline H.sapiens & GAAACGCCCC-GCCGGGCAGGCCGCACCAATGGACGCCGAGCTCGGCCGTGCGTCACGCG \\
\hline M.mulatta & GATACGCCCC-GCCGGGCAGGCCGGGCCAATGGACGCCGAGCTCGGCCGTGCGTCACGCG \\
\hline E.caballus & GCCCCGCCCCTGCCGGGCCCGGCGGGCCAATGGGAACCGAGATCGGCTGTGCGTCACGC \\
\hline B. taurus & CCCCGCCOCC-TCAGGATCCGCCGQGCCAATGGGCGCAGAGGTAGGCT-TGCGTCACGAG \\
\hline M. musculus & TGAGCCCGCC-CCCGGGACTACAGGACCAATAAAGTGATGAATATACCCGCGCGTCACGGA \\
\hline H.sapiens & ACGCTGGCCAATCGCGGAGGGCACGACCGTAGAAAGGCCGGGCGCGGCGAGGCTGGG-C \\
\hline M.mulatta & ACGCTGGCTAATCGCGGAGGGCCACGACCGTAGAAAGGCCGGGCGCGTCTGGGCTGAC-G \\
\hline E.caballus & GCGCTGGCCAATCGCGGAGGGOCACGATT GTAGAAAGGCTGGGGGTGGCGGGGGCGGCCG \\
\hline B.taurus & GCGCTGGCCAATCGAGAAGGGOCACGATCGTAGAAAGGCTGGGCCCGGCGGGGCCGGC-C \\
\hline M. musculus & $\begin{array}{l}\text { GCACCGOCCAATCGCGGACGGCCACGACCCTAGAAAGGCTGGGCGCG-- GCAGGA-G } \\
\text { ERSE }\end{array}$ \\
\hline H.sapiens & G-CTGGGCGGCTGCGGCGCGCGGTGCGCGGTGCGTAGTCTGGAGCTATGGTGGTGGTGGC \\
\hline M.mulatta & G-CTGGGCGGCGGCGGCG-GCGGCGCGCGGTGCGTAGTCTCGAGCTATGGTGGTGGTGGC \\
\hline E.caballus & G-CAGGGCAGCGGCGGCG-CGCAATCGCGTAGAC-AGTCTCTGGCCATGGTGGTGGTGGC \\
\hline B. taurus & CACTGGGCAGCGGCGGCG-CGCACTGGCGTAGACACGCTCCG-GCCAT \\
\hline M. musculus & -CCACGGGGCGGTGGCG-GCGCTGGCGTAGACGTTTCCTGG--CTATGGTGGTG \\
\hline
\end{tabular}

(C)

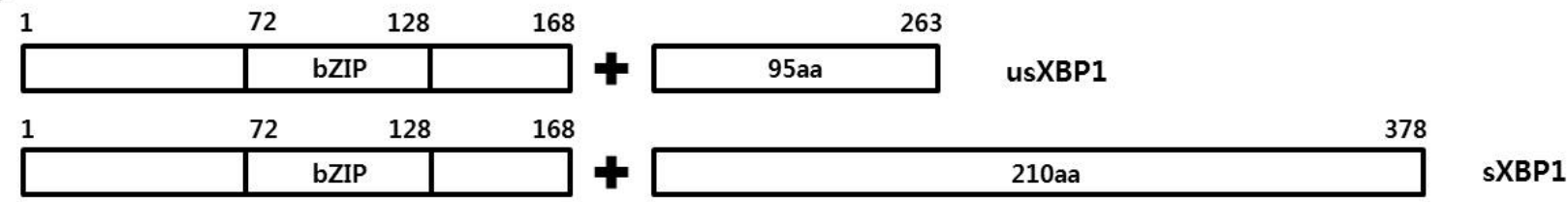

Figure 3. Sequence conservation of XBP1. (A) Protein sequence homology of $s$ XP1 and usXBP1. The values in the matrix indicate the similarity of protein sequences between the two species. (B) Alignment of promoter sequences between species. An arrow indicates the transcription start site $(+1)$ of $X B P 1$. Boxes denote the conserved binding sites of transcription factors in the XBP1 promoter region. Bolded sequences indicate the binding sequences of nuclear factor (NF-Y) (CCAAT), activating transcription factor 6 (ATF6), and XBP1 (CCACG). Putative binding sites of transcription factors are underlined. (C) Schematic representation of the structure of usXBP1 and sXBP1 with amino acid number. The protein length of sXBP1 is longer than that of usXBP1. XBP1, X-box binding protein 1; sXBP1, spliced XBP1; usXBP1, un-spliced XBP1; Pit-1a, POU class 1 homeobox 1; ATF, activating transcription factor; bZIP, basic leucine zipper domain; aa, amino acid.

tains both ERSE and ERSE-II in its promoter region [13]. Thus, Herpud1 is likely induced by various ER-stress reagents [14]. We thus investigated both ERSE and ERSE-II present in horse BiP, GRP94, and Herpud1 promoters. Horse BiP and GRP94 contain ERSE in a similar position to XBP1. Horse GRP94 has an additional version of ERSE but in the reverse direction. In the horse Herpud1 promoter, we also found evidence of ERSE-II (Figure 4A). Unlike humans, horse Herpud1 does not contain ERSE. To understand heat mediated transcription of genes downstream of XBP1, we conducted real-time qPCR of genes containing ERSE, BiP, and GRP94 in their promoter regions. We found that both $\mathrm{BiP}$ and GRP94 transcription were not significantly changed after the $1 \mathrm{~h}$ heat shock, but after the $4 \mathrm{~h}$ heat shock BiP and GRP94 transcription was significantly increased (Figure 4B). These results indicate that the expression of both BiP and GRP94 is affected by the duration of heat stress.

It was initially assumed that UPRE was located in the promoter of ERAD genes such as E3 ubiquitin-protein ligase synoviolin (HRD1) and ER degradation enhancing alphamannosidase-like protein 1 (EDEM1) [15,16]. However, it was later confirmed that the human HRD1 promoter contains functional versions of the UPRE, UPRE-II, and ERSE [17]. However, the binding site of XBP1 in the EDEM1 promoter has not yet been studied. We could not find any evidence of UPRE being present in the horse HRD1 and EDEM1 pro- moter regions. Additionally, even though $H R D 1$ and EDEM1 are solely regulated by the IRE1 $\alpha-X B P 1$ pathway $[16,18]$, UPRE-II and ERSE were only conserved in horse HRD1. Similar to studies of human EDEM1, we could not find any ERstress related elements in the horse EDEM1 promoter (Figure $4 \mathrm{~A})$. As ER-stress induced protein degradation is associated with the IRE1 $\alpha$-XBP1 pathway, we investigated the transcriptional induction of the ERAD-related genes, HRD1 and EDEM1. The expression of both HRD1 and EDEM1 after both $1 \mathrm{~h}$ and $4 \mathrm{~h}$ of heat shock was not altered significantly (Figure $4 \mathrm{C})$. These results suggest that neither $1 \mathrm{~h}$ nor $4 \mathrm{~h}$ of heat shock are sufficient to induce the ERAD system.

According to a recent study, the human citrate carrier $(\mathrm{CiC})$ gene contains UPRE [19]. We searched the horse CiC homologue using the BLAST search tool. Horse clathrin heavy chain 2 isoform 6 (XP_023502409.1), also called solute carrier family 25 member 1 (SLC25A1), showed the highest protein sequence similarity to human CiC (query cover, 99\%; identity, 97\%). We also found that horse SLC25A1 contains UPRE in the promoter region (Figure 4A).

These results clearly indicate that conserved XBP1 binding elements also exist in the horse promoter regions of genes downstream of XBP1. Additionally, as the transcriptional regulation of BiP, GRP94, HRD1, and EDEM1 depends on the duration of heat stress, we can confirm that the refolding and degradation of unfolded and misfolded proteins is stress- 
(A)

\begin{tabular}{|c|c|}
\hline \multirow{3}{*}{$\mathrm{BiP}$} & -307 \\
\hline & GTATCGTCTTCGCCAATCGGCGGCCTCCACGACGGGGCTGGGGGAG \\
\hline & -300 \\
\hline \multirow[t]{2}{*}{ GRP94 } & GGCTACAGATGACCAATCGGACGGAGCCACGCTTCGGGCATCGGGC \\
\hline & $-196 \quad-178$ \\
\hline \multirow[t]{2}{*}{ GRP94 } & GTAGAGGGGGTGCGTGGTGTGGGACGATTGGTGGGTTCGTGTTTCC \\
\hline & $-228 \quad-218$ \\
\hline \multirow[t]{2}{*}{ Herpud1 } & CACGGGTCCGGGCGCCGATTGGGCCACGTTGGGGCAGTGGCCTCCG \\
\hline & $-1482 \quad-1464$ \\
\hline HRD1 & $\frac{\text { TATTGGTCCGCGTAACTCTATCTTGACCAATCAGCAGCAGCCACGG }}{\text { UPRE- II }}$ \\
\hline SLC25A1 & GCCGTGCTCTTAATTGGCTGACGTGGGAGTGGAGAGTTAGTGTCCT \\
\hline
\end{tabular}

(B)

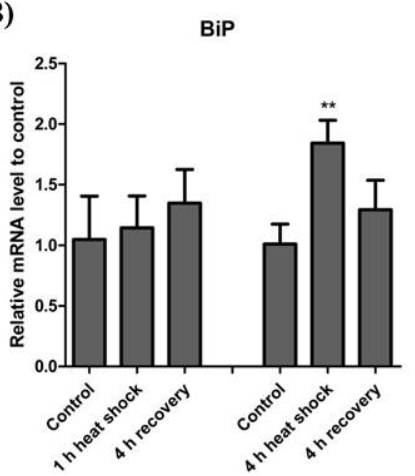

GRP94

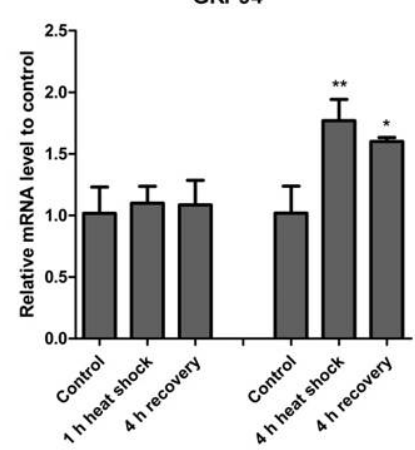

(C)
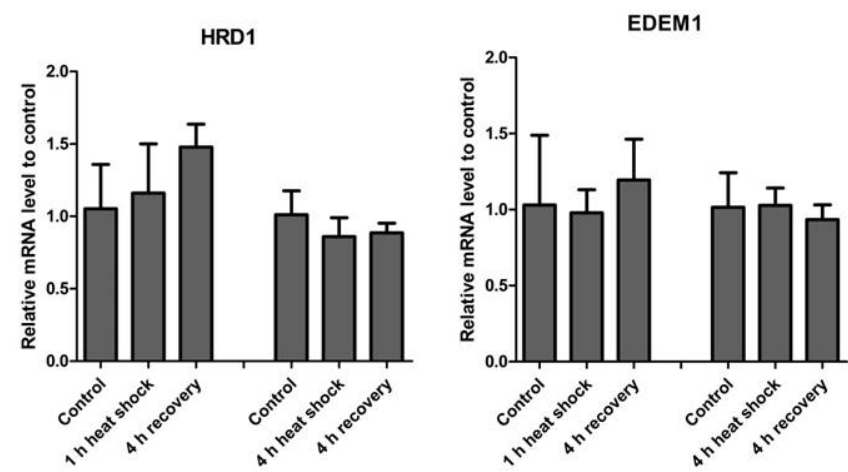

Figure 4. Expression of the downstream genes of XBP1 after heat shock exposure. (A) Conserved binding sequences of the ERSE and ERSE-II on BiP, GRP94, and Herpud1 promoter. The numbers above the sequences denote the position of the transcription start site of each gene. Bolded sequences indicate the binding sequences of nuclear factor (NF-Y) (CCAAT and ATTGG), activating transcription factor 6 (ATF6), and XBP1 (CCACG and CGTGG). (B) Expression of BiP and GRP94 after heat shock. BiP (one-way ANOVA, $1 \mathrm{~h}$ heat shock: $p=0.5033,4 \mathrm{~h}$ heat shock: $p=0.0062$ ) and GRP94 (one-way ANOVA, 1 h heat shock: $p=0.8463,4 \mathrm{~h}$ heat shock: $p=0.0031$ ) expression was investigated using real-time qPCR. (C) Expression of HRD1 (one-way ANOVA, $1 \mathrm{~h}$ heat shock: $p=0.4513,4 \mathrm{~h}$ heat shock: $p=0.3608$ ) and EDEM1 (one-way ANOVA, $1 \mathrm{~h}$ heat shock: $p=0.7198,4 \mathrm{~h}$ heat shock: $p=0.7406$ ) were investigated by real-time $\mathrm{qPCR}$. The results were normalised to GAPDH. Error bars represent the standard deviation ( $n=3)$. XBP1, X-box binding protein 1; ERSE, endoplasmic reticulum-stress response element; BiP, binding immunoglobulin protein; GRP94, heat shock protein $90 \mathrm{kDa}$ beta member 1; Herpud1, homocysteine-responsive endoplasmic reticulum-resident ubiquitin-like domain member 1 protein; UPRE, UPR element; ANOVA, analysis of variance; qPCR, quantitative real-time polymerase chain reaction; HRD1, E3 ubiquitin-protein ligase synoviolin; EDEM1, endoplasmic reticulum degradation enhancing alpha-mannosidase-like protein 1; SLC25A1, solute carrier family 25 member 1.

and/or time-dependent.

\section{DISCUSSION}

ER-stress induces a signalling network known as the UPR which mitigates the negative effects of ER-stress and works to maintain homeostasis. Recent research indicates that the UPR plays important roles in the physiological responses to various diseases, including neurological diseases [20], diabetes [21], cancer [22], and rheumatoid arthritis [23]. ER-stress is induced by a variety of external and environmental factors such as starvation, ischemia, hypoxia, oxidative stress, and heat stress.

Under the heat stress, Hsp72 functions as a molecular chaperone [24]. Exposure to $4 \mathrm{~h}$ of heat shock dramatically increased $h s p 72 \mathrm{mRNA}$ expression levels, and we can thus assume that the duration of heat stress affects the quantity of misfolded and unfolded proteins (Figure 1A). The mRNA expression level of $h s p 72$ was reduced after $4 \mathrm{~h}$ of recovery at $37^{\circ} \mathrm{C}$. This is due to the presence of hsp 72 protein at high concentrations resulting from the $4 \mathrm{~h}$ of heat shock. Conversely, spliced XBP1 mRNAs were maintained at high concentrations even after $4 \mathrm{~h}$ of recovery in the $4 \mathrm{~h}$ heat shock treatment (Figure 1C, 2C). The prolonged persistence of spliced XBP1 may be facilitated by IRE1 $\alpha-X B P 1$ signalling and the XBP1 self-regulatory system $[8,25]$ (Figure $3 B$ ).

When the IRE1 $\alpha-\mathrm{XBP} 1$ pathway is induced in response to ER-stress, sXBP1 regulates downstream genes such as $B i P$, GRP94, Herpud1, and HRD1 [7,13,17]. ERSE, ERSE-II, UPRE, and UPRE-II have been identified to date [15,17], and our result shows that horse ER stress-related genes contain conserved ERSE, ERSE-II, UPRE, and UPRE-II sequences (Figure $4 A$ ). It was postulated that ERAD genes contain UPRE in their promoter regions. A partially palindromic sequence (T $\underline{\mathrm{GAC}}$ GTGG/A) in ATF6a was initially found by binding site selection experiments [26]. However, it has been suggested that the consensus sequence of the ATF6 $a$ binding site in its UPRE is not for ATF6a but for XBP1 [3]. It has also been suggested that ATF6 $\alpha$ produced at physiologically accurate concentrations is insufficient to induce the transactivation of the UPRE reporter due to its low affinity for the UPRE [15]. Interest- 
ingly, although UPRE has been intensively studied in yeast [27,28], UPRE was not found in mammalian promoter regions for a considerable time [15]. Recently, however, UPRE was found in the promoter region of the human $\mathrm{CiC}$ gene [19]. In this study, we located the UPRE sequence in horse SLC25A but not in ERAD genes such as HRD1 and EDEM1. Therefore, it is likely that other ER-stress-related elements may compensate for the absence of UPRE in horse ERAD genes or perhaps a novel and unique mechanism exists. The differences between species in their functional ER-stress-related elements, including UPRE, should be studied in greater detail in the future.

As XBP1 mRNA is spliced after ATF6a cleavage, the UPR undergoes a time-dependent transition from the 'refolding only' phase to the 'refolding plus degradation' phase [16]. Although XBP1 downstream genes contain consensus binding elements, their induction times differ according to their function. For example, EDEM shows increased delayed transcriptional induction when compared to BiP. Mouse embryonic fibroblasts devoid of IRE1 a demonstrate that EDEM mRNA is not produced under ER-stress. Considering that physiologically accurate concentrations of ATF6a could not induce UPRE, UPRE is likely solely regulated by the IRE1 $\alpha-X B P 1$ pathway [16]. Although horse HRD1 and EDEM1 do not contain UPRE, their expression was not altered to the same degree as BiP and GRP94, suggesting that $4 \mathrm{~h}$ of heat shock is not a sufficient duration to induce the ERAD system (Figure 4C). Therefore, our findings support a time-dependent transition model. In the future, the progression of the IRE1 $\alpha$ XBP1 pathway and the level of gene expression relative to the duration and intensity of heat stress should be investigated.

In conclusion, we firstly investigated ER-stress in horse muscle cells subjected to mild heat stress and verified the existence of frame shift splicing in horse XBP1. We also confirmed that the genes related to the UPR and other associated regulatory elements were highly conserved among different animal groups. Our results provide valuable information for the continuation of ER stress-related studies in horse in relation to various environmental stresses.

\section{CONFLICT OF INTEREST}

We certify that there is no conflict of interest with any financial organization regarding the material discussed in the manuscript.

\section{ACKNOWLEDGMENTS}

This work was supported by a grant from the Next-Generation BioGreen 21 Program (Project No. PJ01325701), the Rural Development Administration, Republic of Korea, and a grant from the Individual Basic Science \& Engineering Research
Program (2017R1D1A1B03036432), the National Research Foundation of Korea (NRF), and the Korean government.

\section{REFERENCES}

1. Wu J, Kaufman RJ. From acute ER stress to physiological roles of the unfolded protein response. Cell Death Differ 2006;13: 374-84. https://doi.org/10.1038/sj.cdd.4401840

2. Calfon M, Zeng $\mathrm{H}$, Urano F, et al. IRE1 couples endoplasmic reticulum load to secretory capacity by processing the XBP-1 mRNA. Nature 2002;415:92-6. https://doi.org/10.1038/415092a

3. Yoshida H, Matsui T, Yamamoto A, Okada T, Mori K. XBP1 mRNA is induced by ATF 6 and spliced by IRE1 in response to ER stress to produce a highly active transcription factor. Cell 2001;107:881-91. https://doi.org/10.1016/S0092-8674(01) 00611-0

4. Uemura A, Oku M, Mori K, Yoshida H. Unconventional splicing of XBP1 mRNA occurs in the cytoplasm during the mammalian unfolded protein response. J Cell Sci 2009;122:287786. https://doi.org/10.1242/jcs.040584

5. Mori K. Frame switch splicing and regulated intramembrane proteolysis: key words to understand the unfolded protein response. Traffic 2003;4:519-28. https://doi.org/10.1034/j.16000854.2003.00112.x

6. Roy B, Lee AS. The mammalian endoplasmic reticulum stress response element consists of an evolutionarily conserved tripartite structure and interacts with a novel stress-inducible complex. Nucleic Acids Res 1999;27:1437-43. https://doi.org/ 10.1093/nar/27.6.1437

7. Yoshida H, Haze K, Yanagi H, Yura T, Mori K. Identification of the cis-acting endoplasmic reticulum stress response element responsible for transcriptional induction of mammalian glucose-regulated proteins. Involvement of basic leucine zipper transcription factors. J Biol Chem 1998;273:33741-9. https:// doi.org/10.1074/jbc.273.50.33741

8. Yoshida H, Okada T, Haze K, et al. ATF6 activated by proteolysis binds in the presence of NF-Y (CBF) directly to the cisacting element responsible for the mammalian unfolded protein response. Mol Cell Biol 2000;20:6755-67. https://doi.org/10. 1128/MCB.20.18.6755-6767.2000

9. Marlin DJ, Scott CM, Roberts CA, Casas I, Holah G, Schroter RC. Post exercise changes in compartmental body temperature accompanying intermittent cold water cooling in the hyperthermic horse. Equine Vet J 1998;30:28-34. https://doi. org/10.1111/j.2042-3306.1998.tb04085.x

10. Livak KJ, Schmittgen TD. Analysis of relative gene expression data using real-time quantitative PCR and the 2(-Delta Delta C(T)) method. Methods 2001;25:402-8. https://doi.org/10. 1006/meth.2001.1262

11. Kearse M, Moir R, Wilson A, et al. Geneious Basic: an integrated and extendable desktop software platform for the organization and analysis of sequence data. Bioinformatics 2012;28:1647- 
9. https://doi.org/10.1093/bioinformatics/bts199

12. Xu X, Gupta S, Hu W, McGrath BC, Cavener DR. Hyperthermia induces the ER stress pathway. PLoS One 2011;6:e23740. https://doi.org/10.1371/journal.pone.0023740

13. Kokame K, Kato H, Miyata T. Identification of ERSE-II, a new cis-acting element responsible for the ATF6-dependent mammalian unfolded protein response. J Biol Chem 2001;276:9199205. https://doi.org/10.1074/jbc.M010486200

14. Kokame K, Agarwala KL, Kato H, Miyata T. Herp, a new ubiquitin-like membrane protein induced by endoplasmic reticulum stress. J Biol Chem 2000;275:32846-53. https://doi.org/10. 1074/jbc.M002063200

15. Yamamoto K, Yoshida H, Kokame K, Kaufman RJ, Mori K. Differential contributions of ATF6 and XBP1 to the activation of endoplasmic reticulum stress-responsive cis-acting elements ERSE, UPRE and ERSE-II. J Biochem 2004;136:343-50. https:// doi.org/10.1093/jb/mvh122

16. Yoshida H, Matsui T, Hosokawa N, Kaufman RJ, Nagata K, Mori K. A time-dependent phase shift in the mammalian unfolded protein response. Dev Cell 2003;4:265-71. https:// doi.org/10.1016/S1534-5807(03)00022-4

17. Yamamoto K, Suzuki N, Wada T, et al. Human HRD1 promoter carries a functional unfolded protein response element to which XBP1 but not ATF6 directly binds. J Biochem 2008;144:47786. https://doi.org/10.1093/jb/mvn091

18. Kaneko M, Ishiguro M, Niinuma Y, Uesugi M, Nomura Y. Human HRD1 protects against ER stress-induced apoptosis through ER-associated degradation. FEBS Lett 2002;532:14752. https://doi.org/10.1016/S0014-5793(02)03660-8

19. Damiano F, Tocci R, Gnoni GV, Siculella L. Expression of citrate carrier gene is activated by ER stress effectors XBP1 and ATF6alpha, binding to an UPRE in its promoter. Biochim Biophys Acta Gene Regul Mech 2015;1849:23-31. https:// doi.org/10.1016/j.bbagrm.2014.10.004

20. Hoozemans JJ, Veerhuis R, Van Haastert ES, et al. The unfolded protein response is activated in Alzheimer's disease. Acta
Neuropathol 2005;110:165-72. https://doi.org/10.1007/s00401005-1038-0

21. Ozcan U, Cao Q, Yilmaz E, et al. Endoplasmic reticulum stress links obesity, insulin action, and type 2 diabetes. Science 2004; 306:457-61. https://doi.org/10.1126/science.1103160

22. Dong D, Ni M, Li J, et al. Critical role of the stress chaperone GRP78/BiP in tumor proliferation, survival, and tumor angiogenesis in transgene-induced mammary tumor development. Cancer Res 2008;68:498-505. https://doi.org/10.1158/00085472.CAN-07-2950

23. Yamasaki S, Yagishita N, Tsuchimochi K, Nishioka K, Nakajima T. Rheumatoid arthritis as a hyper-endoplasmic-reticulumassociated degradation disease. Arthritis Res Ther 2005;7:181. https://doi.org/10.1186/ar1808

24. Mayer MP, Bukau B. Hsp70 chaperones: cellular functions and molecular mechanism. Cell Mol Life Sci 2005;62:670. https://doi.org/10.1007/s00018-004-4464-6

25. Lee K, Tirasophon W, Shen X, et al. IRE1-mediated unconventional mRNA splicing and S2P-mediated ATF6 cleavage merge to regulate XBP1 in signaling the unfolded protein response. Genes Dev 2002;16:452-66. https://doi.org/10.1101/ gad.964702

26. Wang Y, Shen J, Arenzana N, Tirasophon W, Kaufman RJ, Prywes R. Activation of ATF6 and an ATF6 DNA binding site by the endoplasmic reticulum stress response. J Biol Chem 2000;275:27013-20.

27. Cox JS, Walter P. A novel mechanism for regulating activity of a transcription factor that controls the unfolded protein response. Cell 1996;87:391-404. https://doi.org/10.1016/S00928674(00)81360-4

28. Mori K, Ogawa N, Kawahara T, Yanagi H, Yura T. Palindrome with spacer of one nucleotide is characteristic of the cis-acting unfolded protein response element in Saccharomyces cerevisiae. J Biol Chem 1998;273:9912-20. https://doi.org/10.1074/jbc. 273.16.9912 